\title{
HIGH-RESOLUTION RHYTHMOCARDIOGRAPHY IN CLINICAL NEUROCARDIOLOGY
}

\author{
V. A. Mironov ${ }^{1 *}$, T. F. Mironova ${ }^{2}$, A. N. Andreev ${ }^{1}$ \\ ${ }^{1}$ Urals State Medical University, 3 Repina St., 620028, Ekaterinburg, Russia \\ ${ }^{2}$ Federal State Center of Cardiovascular Surgery, 2 Geroya Rossii Rodionova E. N. Ave., 454003, Chelyabinsk, Russia \\ *Corresponding author. E-mail: vamironov2013@yandex.ru \\ Address for correspondence: 3 Repina ul., 620028, Ekaterinburg, Russia
}

The paper presents an innovative diagnostic computer complex for heart rate variability (HRV) analysis in clinical neurocardiology. It considers the advances and prospects of the application of highresolution rhythmocardiography (RCG) in the practical medicine, as well as the already obtained results of RCG used in medicine to reveal HRV dysregulation in different nosological diseases.

Keywords: clinical neurocardiology, rhythmocardiography, advances, prospects.

DOI: $10.17804 / 2410-9908.2017 .3 .023-035$

\section{References}

1. Antyufiev V.F., Mironova T.F., Mironov V.A. Disfunktsiya sinoatrialnogo uzla serdtsa [Sinoatrial Node Dysfunction]. Chelyabinsk, Rekpol Publ., 2009, 197 p. (In Russian).

2. Voropaeva E.E. Samoproizvolnyi abort: etiopatogenez, kliniko-morfologicheskaya kharakteristika, reabilitatsiya: diss. dokt. med. nauk [Natural Abortion: Etiopathogenesis, ClinicoMorphological Characteristics, Rehabilitation. Doctoral Thesis]. 2011, 449 p. (In Russian).

3. Davydova E.V. Zakonomernosti dizregulyatsii peismeikernoy aktivnosti sinusovogo uzla serdtsa u bolnykh professionalnymi zabolevaniyami: diss. dokt. med. nauk [Regularities in the Dysregulations of the Pacemaker Activity of the Sinoatrial Node of Patients with Professional Diseases. Doctoral Thesis]. Irkutsk, 2011, 324 p. (In Russian).

4. Zhemaitite D.I. Vegetative regulation and development of CHD complications. Fiziologiya Cheloveka, 1989, no. 15 (2), pp. 3-13. (In Russian).

5. Kassil G.N. Vegetative regulation of internal environment homeostasis. In: Fiziologiya vegetativnoy nervnoy sistemy [Physiology of Vegetative Nervous System]. Leningrad, Nauka Publ., 1981, pp. 536-572. (In Russian).

6. Kuvatov V.A., Mironov V.A., Bavykin M.V., Mironova T.F. Heart rate variability of CHD patients at CABG. Vestnik Udmurtskogo Universiteta. Seriya "Biologiya. Nauki o Zemle”, 2012, iss. 2, pp. 68-78. (In Russian).

7. Mironova T.F., Mironov V.A. Variabelnost serdechnogo ritma pri ishemicheskoy bolezni serdtsa [Heart Rate Variability at Coronary Heart Disease]. Chelyabinsk, Rekpol Publ., 2008, 173 p. (In Russian).

8. Mironova T.F., Mironov V.A. Klinicheskiy analiz volnovoy struktury sinusovogo ritma serdtsa. [Clinical Analysis of the Wave Structure of the Sinoatrial Rate]. Chelyabinsk, Chelyabinskiy Rabochiy Publ., 1998, 162 p. (In Russian).

9. Mironova T.F. Dizregulyatornye vegetativnye rasstroistva kardiovaskulyarnoy sistemy $v$ klinike $i$ patogeneze vibratsionnoy bolezni: diss. dokt. med. nauk [Dysregulatory Vegetative Cardiovascular System Disorders in the Clinical Picture and Pathogenesis of Vibration Disease. Doctoral Thesis]. St. Petersburg, 1990, 507 p. (In Russian).

10. Mironov V.A. Klinicheskiy analiz volnovoy struktury sinusovogo ritma serdtsa pri gipertonicheskoy bolezni: avtoref. dis. dokt. med. nauk [Clinical Analysis of the Wave Structure of the Sinoatrial Rhythm at Arterial Hypertension. Doctoral Thesis Abstract]. Orenburg, 1999, 54 p. (In Russian). 
11. Mironov V.A. Ritmokardiograficheskoe issledovanie perifericheskoy vegetativnoy regulyatsii peismeikernoy aktivnosti sinusovogo uzla pri ishemicheskoy bolezni serdtsa: dis. kand. med. nauk [Rhythmo-Cardiographic Study of the Peripheral Vegetative Regulation of the Sinoatrial Node Pacemaker Activity at Coronary Heart Disease. Cand. Thesis]. Chelyabinsk, 2002, $137 \mathrm{p}$. (In Russian).

12. Sadyrin A.V. Immunologicheskie $i$ vegetativnye narusheniya u bolnykh s tserebralnymi opukholyami pri luchevoy terapii: dis. kand. med. nauk [Immunological and Vegetative Disorders in Cerebral Tumor Patients during Radiotherapy. Cand. Thesis]. Chelyabinsk, 2008, 208 p. (In Russian).

13. Solovieva O.V. Osobennosti perifericheskoy vegetativnoy regulyatsii sinusovogo uzla serdtsa u bolnykh sistemnoy krasnoy volchankoy: diss. kand. med. nauk [Specific Features of Peripheral Vegetative Regulation of the Sinoatrial Node in Patients with Systemic Lupus Erythematosus. Cand. Thesis]. Perm, 2011, 144 p. (In Russian).

14. Tyurin A.Yu. Osobennosti perifericheskoy vegetativnoy regulyatsii peismeikernoy aktivnosti sinusovogo uzla serdtsa u bolnykh s ostrym infarktom miokarda: diss. kand. med. nauk [Specific Features of Peripheral Vegetative Regulation of the Sinoatrial Node Pacemaker Activity in Patients with Acute Myocardial Infarction. Cand. Thesis]. Perm, 2005, 160 p. (In Russian).

15. Shadrina I.M. Perifericheskie vegetativnye effecty nebivolola i bisoprolola po dannym analiza volnovoy variabelnosti serdechnogo ritma pri gipertonicheskoy bolezni: dis. kand. med. nauk [Peripheral Vegetative Effects of Nebivolol and Bisoprolol According to the Analysis of Heart Rate Wave Variability at Arterial Hypertension. Cand. Thesis]. Chelyabinsk, 2010, $178 \mathrm{p}$. (In Russian).

16. Akselrod S. Components of heart rate variability. Basic studies. In: Heart Rate Variability, M. Malik, A.J. Camm, eds., Armonk, NY, Futura Publishing Company Inc., 1995, pp. 147-163.

17. Baselli G., Cerutti S., Civardi S., Liberati D., Lombardi F., Malliani A., Pagani M. Spectral and cross-spectral analysis of heart rate and arterial blood pressure variability signals. Comp. Biomed. Res., 1986, vol. 19, pp. 520-534.

18. Bosner M.S., Kleiger R.E. Heart rate variability and risk stratification after myocardial infarction. In: Heart Rate Variability, M. Malik, A.J. Camm, eds., NY, Futura Publishing Company Inc., 1995, pp. 331-340.

19. Brown D.R., Randall D.C., Knapp C.F., Lee K.C., Yingling J.D. Stability of the heart rate power spectrum over time in the conscious dog. FASEB J, 1989, vol. 3, no. 5, pp. 1644-1650.

20. Camm A.J. Risk stratification following myocardial infarction: heart rate variability and other risk factors. Heart Rate Variability, M. Malik, A.J. Camm, eds., Armonk, NY, Futura Publishing Company Inc., 1995, pp. 369-392.

21. Chess G.F., Tam R.M.K., Calaresu F.R. Influence of cardiac inputs on rhythmic variations of heart period in the cat. Am. J. Physiol., 1975, vol. 220, no. 3, p. 775-780.

22. Fei L., Copie X., Malik M., Camm A.J. Short- and long-term assessment of heart rate variability for risk stratification after acute myocardial infarction. Am. J. Cardiol., 1996, vol. 77, pp. 681-684.

23. Furlan R., Guzzetti S., Crivellaro W., Dassi S., Tinelli M., Baselli G., Cerutti S., Lombardi F., Pagani M., Malliani A. Continuous 24-hour assessment of the neural regulation of systemic arterial pressure and RR variabilities in ambulant subjects. Circulation, 1990, vol. 81, pp. 537-547. DOI: 10.1161/01.CIR.81.2.537.

24. Hainsworth R. The control and physiological importance of heart rate. In: Heart Rate Variability, M. Malik, A.J. Camm, eds., Armonk, NY, Futura Publishing Company Inc., 1995, pp. 21-30. 25. Kautzner J., Fiala M., Hnatkova K. et al. Influence of autonomic modulation of the atrioventricular junction on the heart rate variability measured from R-R intervals. In: Abstracts of XIX Congress of the ESC, Stockholm, Sweden, 1997, pp. 731.

26. Kitney R.J., Rompelman O. The study of Heart Rate Variability, Oxford, England, Claredon Press, 1980. 
27. Kleiger Robert E., Miller J. Philip, Bigger Jr. J. Thomas, Moss Arthur J. Decreased heart rate variability and its association with increased mortality after acute myocardial infarction. Am. J. Cardiol., 1987, vol. 59, pp. 256-262. DOI: 10.1016/0002-9149(87)90795-8.

28. Kleiger R.E, Stein P.K, Bosner M.S, Rottman J.N. Time domain measurements of heart rate variability. Cardiology clinics, 1992, vol. 10, no. 3, pp. 487-98.

29. Levy M.N. Sympathetic-parasympathetic interactions in the heart. Circ. Res., 1971, vol. 29, pp. 437-445.

30. Malik M., Camm A.J. Preface. In: Heart Rate Variability, M. Malik, A.J. Camm, eds., Armonk , NY, Futura Publishing Company Inc., 1995, p. IX.

31. Wall P.D., Melzack R. Visceral versus somatic mechanisms. In: Textbook of Pain, A. Malliani, M. Pagani, F. Lombardi, eds., 2nd ed., Churchill Livingstone, Edinburgh, 1989, pp. $128-140$.

32. Malliani A., Pagani M. , Lombardi F., Cerutti S. Cardiovascular neural regulation explored in the frequency domain. Circulation, 1991, vol. 84, pp. 482-492. DOI: 10.1161/01.CIR.84.2.482.

33. Malliani A. Association of heart rate variability components with physiological regulatory mechanisms. In: Heart Rate Variability, M. Malik, A.J. Camm, eds., Armonk, NY, Futura Publishing Company Inc., 1995, pp. 173-188.

34. Maliani A. Physiological interpretation of the spectral components of heart rate variability (HRV). Vestnik Aritmologii, 1998, no. 9, pp. 7-57. (In Russian).

35. Mancia G., Grassi G., Parati G., Daffonchio A. Evaluating sympathetic activity in human hypertension. J. Hypertens., 1993, vol. 11 (suppl. 5), pp. S13-S19.

36. Mironova T., Mironov V. Clinical Analysis of Heart Rate Variability, Chelyabinsk, 2000, 208 p. (In Russian).

37. Mironov V., Mironova T. Practical Russian experience of using complex apparatus and programs for analysis of heart rate variability. In: The World Congress on Computers in Cardiology, Vienna, Austria, IEEE, Chicago, USA, 1995, pp. 460-472.

38. Pagani M., Lombardi F., Guzzetti S., Rimoldi O., Furlan R., Pizzinelli P, Sandrone G., Malfatto G., Dell'Orto S., Piccaluga E. Power spectral analysis of heart rate and arterial pressure variabilities as a marker of sympatho-vagal interaction in man and conscious dog. Circulation Research, 1986, vol. 59, pp. 178-193. DOI: 10.1161/01.RES.59.2.178.

39. Pagani M., Lucini D., Rimoldi O., Furlan R., Piazza S., Biancardi L. Effect of physical and mental exercise on heart rate variability. In: Heart Rate Variability, M. Malik, A.J. Camm, eds., Armonk, NY, Futura Publishing Company Inc., 1995, pp. 245-266.

40. Penaz J., Roukenz J., Van der Waal H.J. Spectral analysis of some spontaneous rhythms in the circulation. In: Biokybernetic, H. Drischel, N. Tiedt, eds., Leipzig, 1968, pp. 1-233.

41. Penaz J., Honzikova N., Fizer B. Spectral analysis of resting variability of some circulatory parameters in men. Physiologia Bohemoslovaca, 1978, vol. 27, pp. 349-357.

42. Rimoldi O., Pierini S., Ferrari A., Cerutti S., Pagani M., Malliani A. Analysis of short-term oscillations of R-R and arterial pressure in conscious dog. Am. J. Physiol., 1990, vol. 258, no. 4, pp. H967-H976.

43. Sayers B.M. Analysis of heart rate variability. Ergonomics, 1973, vol. 16, iss. 1, pp. 17-32. DOI: $10.1080 / 00140137308924479$.

44. Smyth H.S., Sleight P., Pickering G.W. Reflex regulation of arterial pressure during sleep in men. A quantitative method of assessing baroreflex sensitivity. Circ. Res., 1969, vol. 24, pp. 109-121.

45. Schwartz P.J., De Ferrari G.M. Interventions changing heart rate variability after acute myocardial infarction. In: Heart rate variability, M. Malik, A.J. Camm, eds., Armonk, NY, Futura Publishing Co., 1995, pp. 407-420.

46. Heart rate variability: standards of measurement, physiological interpretation and clinical use. Task Force of the European Society of Cardiology and the North American Society of Pacing and Electrophysiology. Circulation, 1996, vol. 93, pp. 1043-1065. 


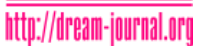

47. Warner H.R., Cox A. A mathematical model of heart rate control by sympathetic and vagus efferent information. J. Appl. Physiol., 1962, vol. 17, pp. 349-355.

48. Zemaityte D., Kesaite R., Varoneckas G. Automic heart control evaluation using longterm recording of RR-intervals. In: Mezhdunarodnyi Simpozium "Kompyuternaya Electrokardiografiya na Rubezhe Stoletiy": tezisy dokladov [International Symposium on Computer Electrocardiography at the Turn of the Centuries, Moscow, April 27-30, 1999.]. M., Kruk Publ., 1999, pp. 120-124. (In Russian). 
Подана в журнал: 31.05 .2017

УДК 616.1/.4 76.29.30.76.13.99

DOI: $10.17804 / 2410-9908.2017 .3 .023-035$

\title{
РИТМОКАРДИОГРАФИЯ ВЫСОКОГО РАЗРЕШЕНИЯ В КЛИНИЧЕСКОЙ НЕЙРОКАРДИОЛОГИИ
}

\author{
В. А. Миронов ${ }^{1 *}$, Т. Ф. Миронова ${ }^{2}$, А. Н. Андреев ${ }^{1}$ \\ ${ }^{1}$ Федеральное государственное бюджетное образовательное учреждение высшего образования \\ «Уральский государственный медицинский университет» Министерства здравоохранения \\ Российской Федерации, ул. Репина, 3, Екатеринбург, Российская Федерация \\ ${ }^{2}$ Федеральное государственное бюджетное учреждение «Федеральный иентр сердечно-сосудистой хирургии» \\ Министерства здравоохранения Российской Федерации, \\ пр. Героя России Родионова Е.Н., 2, Челябинск, Российская Федераџия \\ *Ответственный автор. Электронная почта: vamironov2013@yandex.ru \\ Адрес для переписки: ул. Репина, 3, 620028, Екатеринбург, Российская Федерация
}

В обзоре представлен инновационный диагностический компьютерный комплекс для метода анализа вариабельности сердечного ритма (ВСР) в клинической нейрокардиологии. Обсуждаются достижения и перспективы применения в практической медицине ритмокардиографии высокого разрешения (РКГ), а также уже реализованные в медицинской практике результаты применения РКГ для выявления дизрегуляций ВСР при различных нозологических заболеваниях.

Ключевые слова: клиническая нейрокардиология, ритмокардиография, достижения, перспективы.

\section{1. Введение}

Представлены результаты и некоторые ближайшие перспективы организации и использования в практике инновационного метода ритмокардиографии (РКГ) в диагностике нейрорегуляции при сердечно-сосудистой патологии. Клинические разработки и организация лаборатории нейрокардиологии потребовали длительной научно-исследовательской работы по трем направлениям: 1 - разработке прибора для регистрации ритмокардиограмм, 2 созданию программного обеспечения, 3 - апробации клинического применения высокоточного анализа вариабельности сердечного ритма (ВСР) по нескольким направлениям сравнения результатов РКГ высокого разрешения с данными клинических и параклинических обследований разнопрофильных больных.

В настоящей работе обобщены результаты 24-летнего применения в клинической практике анализа ВСР для оценки разновариантных дизрегуляций синоатриального узла сердца (СУ). Успешные защиты 27 диссертаций, 5 монографий, публикации в России и за рубежом, многочисленные выступления с докладами и, главное, ежедневное применение в практике РКГ убеждают в необходимости использования ВСР-диагностики, её информативности и широких перспективах, как метода ранней диагностики дизрегуляторных кардиоваскулярных нарушений в широком спектре заболеваний. Методологическая основа анализа ВСР и его математическое обеспечение создавались на основе отечественных и зарубежных физиологических исследований $[2,16,17,25,27,30,31,33,34,35,44$ и др.]. Метод, адаптированный к медицинской практике с клинической интерпретацией данных, удалось приоритетно разработать и апробировать с положительным результатом в лечебных учреждениях Урала [37]. Как и стандартно применяемые неспецифические методы (ЭКГ, ХМ, ЭхоКГ) и коронароангиография, РКГ позволяет осуществлять и уточнять интранозологическую диагностику кардиоваскулярной патологии. При этом в большинстве исследований с примене- 
нием РКГ найдены патогенетически обусловленные ВСР-симптомы, в том числе связанные с этиопатогенезом заболевания, т. е. метод является неспецифическим, как и все стандартно применяемые, но по совокупности ВСР-признаков он позволяет определить направление диагностического поиска.

РКГ основана на высокоразрешающем анализе волновой изменчивости продолжительности межсистолических интервалов ритма сердца. Основная сократительная деятельность сердца, потенцируемая автоматически возбудимыми клетками СУ, регулируется симпатическим, парасимпатическим отделами периферической автономной (вегетативной) системы и гуморально-метаболической средой, обязательно влияющей на ионные токи в мембране пейсмекеров при формировании медленных потенциалов действия в СУ. Необходимым условием для корректной клинической интерпретации является высокая точность дис-

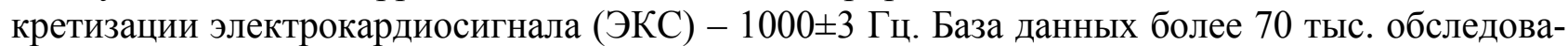
ний больных свидетельствует о необходимости соблюдения такой точности, поскольку нередко ВСР-показатели определяются единицами миллисекунд. В качестве базовой модели был разработан и использовался диагностический комплекс аппаратно-программный (АПК) компьютерной ритмокардиографии КАП-РК-01-«Микор» для коротких 300-интервальных записей, рекомендованный МЗ РФ, Федеральным агентством по надзору в сфере здравоохранения (Рег. удостоверение №ФС 02262005/2447-06, ТУ-9441-002-21569106-2005), с разрешением регистрации ЭКС в 1 миллисекунду, что в 8-14 раз точнее применяемых ЭКГсистем. Запись осуществлялась в строго стационарных контролируемых условиях с дискретизацией ЭКС в 1000 Гц (1000 опросов в 1 с). Необходимость точности съема ЭКС до 1 миллисекунды, хранение с той же точностью в оперативной памяти компьютера и такие же математические расчеты потребовали разработки специального внешнего прибора с рядом схемно-технических решений для усиления и фильтрации ЭКС. Ещё одним условием является корректность программной компьютерной обработки РКГ. Она содержит приемы автоматизированной обработки на 5 стационарных постстимульных участках интервалограмм по 260-300 интервалов в каждой позиции, при 3-5-минутной регистрации с кратным увеличением этого времени в зависимости от числа избранных функциональных нагрузочных проб. Программа состоит из модулей: «Запись РКГ» для регистрации и построения РКГ в реальном текущем времени на экране; «Ввод данных» испытуемого, «Редактирование» с использование 4 компьютерных программ компьютерными способами для исправления или удаления нестационарностей перед анализом, «Анализ» для автоматизированного статистического временного и спектрального анализа волновой структуры ВСР и периодов стимуляции в пробах, «Работа с архивом» для сохранения базы данных и работы с нею, «Заключение» для формализованного диагноза. Эпизоды аритмий и другие нестационарные данные удалялись перед анализом с сохранением первичных и отредактированных данных. Файл содержит 280 формализованных диагностических заключений и другие специальные возможности. По гранту Министерства экономического развития Челябинской области разработана и используется новая модификация АПК с мониторной записью для кардиохирургических операций. Метод, программное обеспечение и комплекс КАП-РК-01 защищены патентами РФ, как интеллектуальная собственность разработчиков.

Физиологической основой РКГ является оценка периферической вегетативной (автономной) симпатической и парасимпатической, а также гуморально-метаболически-медиаторной регуляции ВСР по визуально-логической и математической характеристике трех видов флуктуаций ВСР определенных частоты и периодичности. Они формируются в соответствии в зависимости от скорости проведения импульсов по симпатическим и парасимпатическим волокнам, а также в зависимости от колебаний концентраций активных веществ в тканевых гуморальных биосредах, влияющих на потенциалы действия в пейсмекерных клетках СУ [5]. Программное обеспечение должно учитывать особенности периферической автономной регуляции СУ, особенно в клинической интерпретации данных. 
Результатами экспериментальных работ $[21,24,25,26$, , 20, 34, 43 и др.] доказана физиологическая обусловленность высокочастотных (HF) волн СР преимущественно парасимпатическими отрицательными хронотропными влияниями на пейсмекеры СУ. Это воздействие формируется эфферентными импульсами надсегментарного уровня и, в большей мере, реципрокной передачей по вагусным волокнам раздражения ирритантных, юксткапиллярных и механических рецепторов легочной паренхимы при вдохах-выдохах [11]. Скорость передачи импульсов по парасимпатическим волокнам самая большая, по Warner [48] латентный период раздражения в них - 200 миллисекунд (мс), поэтому отрицательный эффект удлинения фазы диастолической деполяризации при формировании медленных потенциалов действия в СУ успевает реализоваться лишь увеличением 1-2 интервалов, что и образует удлинения RR-интервалов короткого периода - s или в спектральном анализе - HF. Их частота и амплитуда характеризуют преимущественно вагусные влияния в СУ [16, 37].

Впервые волновые колебания артериального давления на ЧСС обнаружены Mayer в 1876 г. Позднее Smyth [44] и Penaz [40, 41] связали эти волны с симпатической и барорецепторной активностью тканевых рецепторов сердца, и ещё позднее $[10,17,23,35,36,38,39$, $42,44]$ в экспериментах и клинических наблюдениях при анализе ВСР доказана связь волн Майера с симпатической активностью и сосудистым тонусом [10]. Период этих волн $-\mathrm{m}$ длиннее и, соответственно, частота ниже из-за медленной (1-3 с) скорости проведения импульсов по симпатическим волокнам [47], поэтому эти флуктуации соотносятся с низкочастотной периодикой CP (0,04-0,15 Гц), называются LF, или «m-волны», и характеризуют симпатические влияния в СУ. Третий вид волн CP-VLF, или «l-волны», с периодом колебаний более 30 до 57с и частотой 0,003-0,04 Гц связан с гуморально-метаболическими влияниями в СУ, участвующими в формировании медленных потенциалов действия в автоматически возбудимых пейсмекерных клетках СУ. Доказательства содержатся в работах лаборатории нейрогуморальной регуляции (МГМУ им. И.П. Сеченова), в которых временные параметры колебаний концентраций активных веществ совпадают с частотными характеристиками 1-волн [7, 8]. Akselrod S. [16, 17] показала связь колебаний BCP менее 0,04 Гц с ангиотензин-рениновой системой. В работах Д. Жемайтите [4] в многолетних обследованиях пациентов с ИБС также прослеживается сопряжённость VLF с гуморально-метаболическими воздействиями на СР. В представляемых исследованиях патология гормонопродуцирующих органов и обмена веществ, как правило, сопровождалась увеличением спектральной мощности $\operatorname{VLF}[5,13]$. Четвертый вид волн - ultra low frequency (ULF), связанных с терморегуляцией, циркадными перепадами и церебральными влияниями на ВСР, в настоящем представлении не рассматривается. По данным Д. Жемайтите и др. [48], сопоставивших диагностическую ценность HF, LF, VLF и ULF волн CP, наибольшая информативность о периферической автономной регуляции содержится в частотных бандах HF, LF, VLF, ультранизкие же частоты (ULF) опосредуются, в конечном итоге, интегральным показателем ЧСC.

Ритмокардиограмма (РКГ) - это графическое изображение последовательного временного ряда межсистолических интервалов в виде отрезков прямой линии, эквивалентных по длине продолжительности пауз между сокращениями сердца. Каждый из отрезков начинается на оси абсцисс с числом интервалов, и продолжается вверх параллельно оси ординат со шкалой времени в секундах (c). Выделяются три диапазона волн ВСР 0,003-0,04; 0,04-0,15; 0,15-0,4 Гц, получивших в зарубежной литературе названия - VLF, LF, HF, т. е. очень низкочастотные, низкочастотные и высокочастотные составляющие энергетического спектра ВСР [46]. В представляемой математической обработке РКГ временной (Тіmе Domain) анализ сочетается со спектральным (Frequency Domain) [7, 8, 30, 32].

В РКГ-исследовании применялись пробы: Вальсальвы-Бюркера (Vm), Ашнера (Pa), активная ортостатическая (Аор) с физической нагрузкой на велоэргометре (PL). РКГ данные в пробах сопоставлялись с фоновой записью в покое лежа $(\mathrm{Ph})$. Выделялись временные статистические средние значения: $R R(\mathrm{NN})$, среднеквадритические отклонения всех волн ВСР $\sigma \mathrm{RR}-(\mathrm{SDNN})$, а также гуморальных, симпатических и парасимпатических волн - $\sigma \mathrm{l}, \sigma \mathrm{m}, \sigma \mathrm{s}$. 
Названия «l-, m-, s-волны» определены продолжительностью периодов этих волн (long, middle, short). ARA - амплитуда дыхательной аритмии - средняя величина всех высокочастотных удлинений интервалов. В частотном анализе выделялись доли мощностей спектральной плотности трех диапазонов в общем спектре колебаний CP, принятом за $100 \%$ - VLF \%, $\mathrm{LF} \%$, HF \%, их доли характеризуют соотношение трех регулирующих факторов в СУ. Периоды стимуляции оценивались по значениям показателей: $\Delta \mathrm{RR}$ - величина максимальной реакции на стимул, выраженная в процентах относительно исхода; tAB и $\operatorname{tr}$-абсолютное время достижения максимальной реакции на стимул и восстановления после неё до $95 \%$ величины исходного среднего интервала (в активной ортопробе - до $75 \%$ ); $\Delta \mathrm{RR}^{\prime}$ - величина минимального интервала при нагрузке в пробе PWC120 в сек. Использовались также нормированные значения РКГ показателей в тестах, т. е. разница между исходными значениями РКГ показателя и его величиной в период стимуляции $(\Delta \sigma \mathrm{l}, \Delta \sigma \mathrm{m}, \Delta \sigma \mathrm{s}, \Delta \sigma \mathrm{l} \%$ и т.д.), нормированная на значения тех же РКГ признаков в исходной позиции-Ph, согласно закона «исходного уровня» и авторских рекомендаций W. Wielder (1957) - nuRR, nu $\sigma \mathrm{RR}, \mathrm{nu} \sigma \mathrm{s}, \mathrm{nu}$ ARA и т. д.

База данных в настоящее время насчитывает более 70 тысяч РКГ-исследований пациентов с различными кардиоваскулярными и экстракардиальными болезнями. РКГ-данные при разработке каждого тематического фрагмента исследований сопоставлялись со здоровым, сопоставимым по возрасту и полу контролем, а также с результатами референсных обследований ЭКГ с велоэргометрическими нагрузками, суточные ЭКГ и АД-мониторирования, ДЭхоКГ и другие инструментальные методы. При оценке по методике В.В. Власова диагностической ценности РКГ-симптомокомплексов дизрегуляции СУ чувствительность РКГ была в пределах 70-87 \%, специфичность 30-45 \%, прогностическая ценность положительного и отрицательного результатов имела разброс от 75 до 88,5 \%. Уровень доказательности - В. Что удалось и доказано на настоящий момент?

1. При аритмиях сердца РКГ позволяет оценить их частоту, время появления в диастоле, определить количество эктопических очагов, дифференцировать желудочковые и наджелудочковые нарушения ритма, парасистолию, скрытую стволовую бигеминию, дисфункцию синусного узла и др. С помощью РКГ в короткой контролируемой записи с применением проб диагностируется более 120 клинических форм кардиоаритмий. А также гемодинамическое значение каждого эпизода, автономный аритмогенный фон неограниченное количество сочетаний патологического аритмогенного фона [1, 9, 10, 36,].

2. Для хронической ИБС характерны РКГ-симптомы - перераспределение регуляции СУ со снижением рефлекторного симпато-парасимпатического влияния (редукция $\sigma \mathrm{m}, \sigma \mathrm{s}, \mathrm{LF} \%, \mathrm{HF} \%)$ и переходом руководства СР на более низкий гуморальный уровень (увеличение VLF \%), снижение и стабилизация ВCР, изменения реагирования на стимулы (снижение $\Delta \mathrm{RR}$, увеличение $\mathrm{tAB}, \mathrm{tr})$. Стенокардия у больных сопровождается выраженной стабилизацией ВСР во время пароксизмов, часто сразу после физической нагрузки. Различия продолжительности соседних RR интервалов находится в пре-

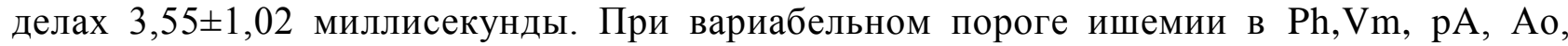
PWC120, т. е. в стационарной части РКГ, появляются участки стабилизации ВСР, в том числе ангиоспастический эпизод ишемии, когда до эпизода или сразу после него усиливается симпатическая периодика $[4,7,8,9,12,14,18,36]$.

3. Субэпикардиальному инфаркту миокарда (ОИМ) в остром периоде соответствовало полное отсутствие волн СР на фоне тахикардии. При субэндокардиальном ОИМ ВСР была более сохранной. В последующем при переднем ИМ снижался уровень ритмокардиограммы, возрастали частота и амплитуда симпатических и гуморальных волн и постепенно увеличивалась величина реакции на стимулы в пробах, что вероятно связано с высокой плотностью адренергических рецепторов в передней стенке левого желудочка сердца. При заднем ОИМ длительно сохранялся ригидный и ареактивный 
ритм, акинезия задней стенки и компенсаторная гиперкинезия передней $[7,11,12,14$, $22,27,45]$. Различия обусловливались асимметрией и неравномерностью интрамурального вегетативного представительства в сердце [33, 34, 44].

4. Воспалительные изменения в миокарде сопровождались формированием высокочастотных непарасимпатических волн (НПВ) из 3-4 удлиненных интервалов и «пиком» спектральной мощности в диапазоне 0,17-0,24 Гц, а также увеличением времени восстановления ВСР после нагрузки. При системной красной волчанке (СКВ) в исследованиях О.В. Соловьёвой, к примеру, у подавляющего большинства обследованных больных с обострением СКВ была такая непарасимпатическая высокочастотная периодика (НПВ) [10, 13].

5. Заболеваниям, сопровождающимся увеличением гормонопродукции, соответствовали повышение реакции в пробе Ашнера и формирование высокоамплитудных гуморальных волн длинного периода. Доказаны, к примеру, РКГ-различия дизрегуляций СУ при диабете 1-го и 2-го типа. При осложнениях диабета воспалительного характера также регистрировались НПВ колебания ВСР. Кроме того, получены доказательства, что волновая структура ВСР ассоциирована с кардиоваскулярной патологией.

6. При сосудистой патологии РКГ позволяет определить особенности ВСР, характерные для артериальной гипертензии, дифференцировать стадии гипертонической болезни, проанализировать фон нарушений регуляции СУ, индивидуально подобрать медикаментозную терапию, проконтролировать её эффективность, в некоторых случаях прогнозировать результат, определить побочные неблагоприятные эффекты $[11,17]$, в отдельных случаях с помощью РКГ оттитровать дозу лекарства, что сочетается с зарубежными исследованиями $[23,32,35,38,39,42]$.

7. При бронхоспастическом синдроме формируются особые волны ВСР в диапазоне VLF, исчезающие при приёме бронходилататоров. Они имеют частоту $38,7 \pm 4,16$ с, «пик» спектральной мощности в очень низкочастотном диапазоне - 0,024 $\pm 0,005$ Гц среднюю и умеренную значимую корреляцию с результатами спирометрии и бодиплетизмографии [3, 38].

8. При профессиональных заболеваниях также найдены дизрегуляции СУ. Вибрационная болезнь сопровождается формированием автономной кардионейропатии с прогрессирующей стабилизацией ВСР $[3,9]$ на фоне тахикардии. Патофизиологически при хроническом действии вибрации нарушается цикличность обменных процессов, в норме сбалансированная с сокращениями сердца. В результате формируется ремоделирование в структурах сердца, в том числе и в узловой ткани, вплоть до автономной кардионейропатии, которая является по признанию ВОЗ предиктором высокого риска летального исхода.

9. При пневмокониозе образование перибронхиального и периваскулярного фиброза в мощной лёгочной паренхиме также ведёт к ремоделированию тканей сердца, лёгочносердечной недостаточности. Это доказано в исследованиях Е.В. Давыдовой [3] с помощью электронной микроскопии тканей миокарда и СУ и сопровождается РКГ-симптомами $[19,29$, $23,26]$. Гистологические исследования свидетельствуют о дистрофии в пейсмекерных клетках СУ.

10. РКГ-исследования у больных с профессиональной интоксикацией соединениями марганца и фтора [3] также позволили выявить дизрегуляцию СУ, соответствующую автономной кардионейропатии. Она сопровождалась нормо- или брадикардией. Эта особенность была обусловлена срывом синтеза норадреналина и адреналина из-за избирательной блокады пиридоксалфосфата при этой интоксикации, а также избытком ионов марганца во внеклеточной среде при формировании потенциалов действия в пейсмекерах СУ.

11. При эндогенной интоксикации у онкологических больных также формируются высокочастотные волны ВСР, отличающиеся от нормальных парасимпатических флуктуаций. Они коррелируют с нарушениями иммунологического статуса у больных (Садырин А.В., 2008) [12]. 
12. Непарасимпатическая периодика - НПВ выявлена при самопроизвольных выкидышах [2]. Это предполагает неманифестированные формы воспалительных изменений репродуктивных органов у женщин.

13. В связи с повышенным интересом к кардиохирургическим операциям за последний год начались исследования возможностей применения РКГ при интервенционных вмешательствах [6]. Создана модификация аппаратно-программного комплекса - КАПРК-02-«Микор» с мониторным режимом записи РКГ для кардиохирургии и дистанционной передачей РКГ-данных из операционного зала для оценки РКГ в реальном текущем времени и консультирования во время операции, продолжаются научно-исследовательские и опытно-конструкторские работы по следующей модификации прибора для отделений реанимации и интенсивной терапии. Оказалось, что каждому этапу операции соответствуют определённые и патогенетически объяснимые изменения волновой структуры ВСР. Найдено, что осложнения во время операции причинно связаны с манипуляциями хирургов во время вмешательства.

14. Интересные и иногда неожиданные данные получены с помощью РКГ в клинической фармакологии [15]. Контроль лечебного воздействия с регистрацией РКГ исходно и после приема лекарственных препаратов позволяет прицельно оценить направленность терапевтической коррекции, прогнозировать её эффект (в некоторых случаях при 1-й разовой дозе), определить побочные действия препаратов. РКГ-контроль с направленными вегетативными тестами при лечении позволил выявить ранее не описанные вегетативные эффекты действия этих лекарств. Кроме того, получены некоторые данные о «независимом» действии лекарственных комплексов при полипрагмазии, что позволяет предвидеть перспективу включения РКГ-исследования в комплекс инструментальных формализованных методов контроля при клинических испытаниях в области фармакологии [15].

Для доказательства каждого фрагмента представляемых результатов выполнены и утверждены Высшей аттестационной комиссией 27 диссертаций и эта работа продолжается. В области создания диагностической аппаратуры перспективы весьма широки. Продолжаются и клинические исследования. Результаты этих работ убеждают, что РКГ адекватный метод и в распознавании дизрегуляторных нарушений и, соответственно, в ранней и интранозологической диагностике РКГ пока не имеет альтернативы.

\section{Литература}

1. Антюфьев В. Ф., Миронова Т. Ф., Миронов В. А. Дисфункция синоатриального узла сердца. - Челябинск : Рекпол, 2009. - 197 с.

2. Воропаева Е. Е. Самопроизвольный аборт: этиопатогенез, клинико-морфологическая характеристика, реабилитация : дисс. ... докт. мед. наук : 14.01.01. - Челябинск, 2011. - 449 с.

3. Давыдова Е. В. Закономерности дизрегуляций пейсмекерной активности синусового узла сердца у больных профессиональными заболеваниями : дисс. ... докт. мед. наук : 14.02.04. - Иркутск, 2011. - 324 с.

4. Жемайтите Д. И. Вегетативная регуляция и развитие осложнений ИБС // Физиология человека. - 1989. - № 15 (2). - С. 3-13.

5. Кассиль Г. Н. Вегетативное регулирование гомеостаза внутренней среды // Физиология вегетативной нервной системы. - Ленинград : Наука, 1981. - С. 536-572.

6. Вариабельность сердечного ритма у больных ишемической болезнью сердца при аортокоронарном шунтировании / В. А. Куватов, В. А. Миронов, М. В. Бавыкин, Т. Ф. Миронова// Вестник Удмуртского университета. Серия «Биология. Науки о Земле». 2012. - Вып. 2. - С. 68-78.

7. Миронова Т. Ф., Миронов В. А. Вариабельность сердечного ритма при ишемической болезни сердца. - 2-е изд., испр. и доп. - Челябинск : Рекпол, 2008. - 173 с. 
8. Миронова Т. Ф., Миронов В. А. Клинический анализ волновой структуры синусового ритма сердца. - Челябинск : Челябинский рабочий, 1998. - 162 с.

9. Миронова Т. Ф. Дизрегуляторные вегетативные расстройства кардиоваскулярной системы в клинике и патогенезе вибрационной болезни : дисс. ... докт. мед. наук : 14.00.05, 14.00.07. - С.-Петербург, 1990. - 507 с.

10. Миронов В. А. Клинический анализ волновой структуры синусового ритма сердца при гипертонической болезни : автореф. дис. ... докт. мед.наук : 14.00.06. - Оренбург, 1999. - 54 с.

11. Миронов М. В. Ритмокардиографическое исследование периферической вегетативной регуляции пейсмекерной активности синусового узла при ишемической болезни сердца : дис. ... канд. мед. наук : 14.00.06. - Челябинск, 2002. - 137 с.

12. Садырин А. В. Иммунологические и вегетативные нарушения у больных с церебральными опухолями при лучевой терапии : дис. ... канд. мед. наук. - Челябинск, 2008. - 208 с.

13. Соловьёва О. В. Особенности периферической вегетативной регуляции синусового узла сердца у больных системной красной волчанкой : дисс. ... канд. мед. наук : 14.01.04. Пермь, 2011. - 144 c.

14. Тюрин А. Ю. Особенности периферической вегетативной регуляции пейсмекерной активности синусового узла сердца у больных с острым инфарктом миокарда : дисс. ... канд. мед. наук : 14.00.06. - Пермь, 2005. - 160 с.

15. Шадрина И. М. Периферические вегетативные эффекты небиволола и бисопролола по данным анализа волновой вариабельности сердечного ритма при гипертонической болезни : дис. ... канд. мед. наук : 14.03.06. - Челябинск, 2010. - 178 с.

16. Akselrod S. Components of heart rate variability. Basic studies // Heart Rate Variability / ed. by M. Malik, A. J. Camm. - Armonk, New York., Futura Publishing Company Inc., 1995. P. 147-163.

17. Spectral and cross-spectral analysis of heart rate and arterial blood pressure variability signals / G. Baselli, S. Cerutti, S. Civardi, D. Liberati, F. Lombardi, A. Malliani, M. Pagani. Comp. Biomed. Res. - 1986. - Vol. 19. - P. 520-534.

18. Bosner M. S., Kleiger R. E. Heart rate variability and risk stratification after myocardial infarction // Heart Rate Variability / ed. by M. Malik, A. J. Camm. - Armonk, New York : Futura Publishing Company Inc., 1995. - P. 331-340.

19. Stability of the heart rate power spectrum over time in the conscious dog / D. R. Brown, D. C. Randall, C. F. Knapp, K. C. Lee, J. D. Yingling. - FASEB J. - 1989. - Vol. 3, no. 5. P. 1644-1650.

20. Camm A. J. Risk stratification following myocardial infarction: heart rate variability and other risk factors // Heart Rate Variability / ed. by M. Malik, A. J. Camm. - Armonk, New York : Futura Publishing Company Inc., 1995. - P. 369-392.

21. Chess G. F., Tam R. M. K., Calaresu F. R. Influence of cardiac inputs on rhythmic variations of heart period in the cat // Am. J. Physiol. - 1975. - Vol. 220, no. 3. - P. 775-780.

22. Short- and long-term assessment of heart rate variabilityfor risk stratification after acute myocardial infarction / L. Fei, X. Copie, M. Malik, A. J. Camm // Am. J. Cardiol. - 1996. Vol. 77. - P. 681-684.

23. Continuous 24-hour assessment of the neural regulation of systemic arterial pressure and RR variabilities in ambulant subjects / R. Furlan, S. Guzzetti, W. Crivellaro, S. Dassi, M. Tinelli, G. Baselli, S. Cerutti, F. Lombardi, M. Pagani, A. Malliani // Circulation. - 1990. - Vol. 81. P. 537-547. - DOI: 10.1161/01.CIR.81.2.537.

24. Hainsworth R. The control and physiological importance of heart rate // Heart Rate Variability / ed. by M. Malik, A. J. Camm. - Armonk, New York : Futura Publishing Company Inc., 1995. - P. 21-30.

25. Influence of autonomic modulation of the atrioventricular junction on the heart rate variability measured from R-R intervals / J. Kautzner, M. Fiala, K. Hnatkova et al. // XIX Annual Congress of the ESC, Stockholm, Sweden : abstracts. - 1997. - P. 731. 
26. Kitney R. J., Rompelman O. The study of Heart Rate Variability. - Oxford, England : Claredon Press, 1980.

27. Decreased heart rate variability and its association with increased mortality after acute myocardial infarction / Robert E. Kleiger, J. Philip Miller, J. Thomas Bigger Jr., Arthur J. Moss // Am. J. Cardiol. - 1987. - Vol. 59. - P. 256-262. - DOI: 10.1016/0002-9149(87)90795-8.

28. Time-Domain measurement of heart rate variability / R. E. Kleiger, P. K. Stein, M. S Bosner, Rottman J. N. // Heart Rate Variability / ed. by M. Malik, A. J. Camm. - Armonk, New York : Futura Publishing Company Inc., 1995. - P. 33-45.

29. Levy M. N. Sympathetic-parasympathetic interactions in the heart // Circ. Res. - 1971. Vol. 29. - P. 437-445.

30. Malik M., Camm A. J. Preface // Heart Rate Variability / ed. by M. Malik, A. J. Camm. Armonk, New York : Futura Publishing Company Inc., 1995. - P. IX.

31. Wall P. D., Melzack R. Visceral versus somatic mechanisms // Textbook of Pain / ed. by A. Malliani, M. Pagani, F. Lombardi et al. - 2-nd ed. - Churchill Livingstone, Edinburgh, 1989. P. 128-140.

32. Cardiovascular neural regulation explored in the frequency domain / A. Malliani, M. Pagani, F. Lombardi, S. Cerutti // Circ. - 1991. - Vol. 84. - P. 482-492. - DOI: 10.1161/01.CIR.84.2.482.

33. Malliani A. Association of heart rate variability components with physiological regulatory mechanisms // Heart Rate Variability / ed by M. Malik, A. J. Camm. - Armonk, New York : Futura Publishing Company Inc., 1995. - P. 173-188.

34. Малиани А. Физиологическая интерпретация спектральных компонентов вариабельности сердечного ритма (HRV) // Вестник Аритмологии. - 1998. - № 9. - С. 7-57.

35. Evaluating sympathetic activity in human hypertension / G. Mancia, G. Grassi, G. Parati, A. Daffonchio // J. Hypertens. - 1993. - Vol. 11 (suppl. 5). - P. S13-S19.

36. Mironova T., Mironov V. Clinical Analysis of Heart Rate Variability. - Chelyabinsk, 2000. -208 p.

37. Mironov V., Mironova T. Practical Russian experience of using complex apparatus and programs for analysis of heart rate variability // The World Congress "Computers in Cardiology", Vienna, Austria. - Chicago, USA : IEEE, 1995. - P. 460-472.

38. Power spectral analysis of heart rate and arterial pressure variabilities as a marker of sympatho-vagal interaction in man and conscious dog / M. Pagani, F. Lombardi, S. Guzzetti, O. Rimoldi, R. Furlan, P. Pizzinelli, G. Sandrone, G. Malfatto, S. Dell'Orto, E. Piccaluga // Circ. Res. - 1986. - Vol. 59. - P. 178-193. - DOI: 10.1161/01.RES.59.2.178.

39. Effect of physical and mental exercise on heart rate variability / M. Pagani, D. Lucini, O. Rimoldi, R. Furlan, S. Piazza, L. Biancardi // Heart Rate Variability / ed. by M. Malik, A. J. Camm. - Armonk, New York : Futura Publishing Company Inc., 1995. - P. 245-266.

40. Penaz J., Roukenz J., Van der Waal H. J. Spectral analysis of some spontaneous rhythms in the circulation // Biokybernetic / ed. by H. Drischel, N. Tiedt. - Leipzig, 1968. - P. 1-233.

41. Penaz J., Honzikova N., Fizer B. Spectral analysis of resting variability of some circulatory parameters in men // Physiologia Bohemoslovaca. - 1978. - Vol. 27. - P. 349-357.

42. Analysis of short-term oscillations of R-R and arterial pressure in conscious dog / O. Rimoldi, S. Pierini, A. Ferrari, S. Cerutti, M. Pagani, A. Malliani // Am. J. Physiol. - 1990. Vol. 258, no. 4. - P. 967-976.

43. Sayers B. M. Analysis of heart rate variability // Ergonomics. - 1973. - Vol. 16, iss. 1. P. 17-32. - DOI: 10.1080/00140137308924479.

44. Smyth H. S., Sleight P., Pickering G. W. Reflex regulation of arterial pressure during sleep in men. A quantitative method of assessing baroreflex sensitivity // Circ. Res. - 1969. - Vol. 24. P. 109-121.

45. Schwartz P. J., De Ferrari G. M. Interventions changing heart rate variability after acute myocardial infarction // Heart rate variability / ed. by M. Malik, A. J. Camm. - Armonk, New York : Futura Publishing Co., 1995. - P. 407-420. 


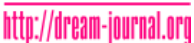

46. Task Force of the European Society of Cardiology and the North American Society of Pacing and Electrophysiology. Heart Rate Variability. Standards of Measurement, Physiological Interpretation, and Clinical Use. - Circulation. - 1996. - Vol. 93. - P. 1043-1065.

47. Warner H. R., Cox A. A mathematical model of heart rate control by sympathetic and vagus efferent information // J. Appl. Physiol. - 1962. - Vol. 17. - P. 349-355.

48. Zemaityte D., Kesaite R., Varoneckas G. Automic heart control evaluation using longterm recording of RR-intervals // Международный симпозиум «Компьютерная электрокардиография на рубеже столетий», Москва, 27-30 апреля, 1999 г. : тезисы докладов. - М. : Крук, 1999. - C. 120-124. 